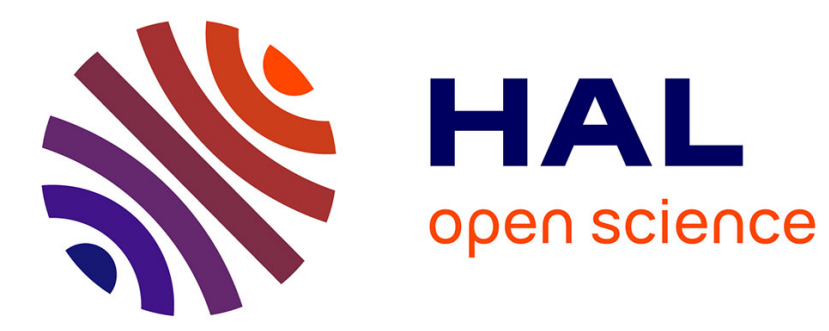

\title{
Effects of Knee Locking and Passive Joint Stiffness on Energy Consumption of a Seven-Link Planar Biped
}

\author{
Abdul Haq, Yannick Aoustin, Christine Chevallereau
}

\section{To cite this version:}

Abdul Haq, Yannick Aoustin, Christine Chevallereau. Effects of Knee Locking and Passive Joint Stiffness on Energy Consumption of a Seven-Link Planar Biped. 2012. hal-00668808

\section{HAL Id: hal-00668808 \\ https://hal.science/hal-00668808}

Preprint submitted on 10 Feb 2012

HAL is a multi-disciplinary open access archive for the deposit and dissemination of scientific research documents, whether they are published or not. The documents may come from teaching and research institutions in France or abroad, or from public or private research centers.
L'archive ouverte pluridisciplinaire HAL, est destinée au dépôt et à la diffusion de documents scientifiques de niveau recherche, publiés ou non, émanant des établissements d'enseignement et de recherche français ou étrangers, des laboratoires publics ou privés. 


\title{
Effects of Knee Locking and Passive Joint Stiffness on Energy Consumption of a Seven-Link Planar Biped
}

\author{
A. Haq, Y. Aoustin and C. Chevallereau
}

\begin{abstract}
Energetic efficiency and stability are the fundamental criteria which can improve the autonomy and task performance capabilities of humanoid robots. The scope of this paper is to investigate the energetic effects of knee locking and addition of torsional springs to different joints of a sevenlink fully actuated planar bipedal robot. The focus is on the reduction of energy consumption during walking. The energetic cost of walking is determined without joint stiffness and knee locking as a baseline for the comparison of results. In the first approach, the gait trajectory is optimized by adding springs to different joints and energetic cost of walk is then calculated at different walking speeds. The second approach presented in this paper is to mechanically lock the support knee and then optimize the gait and calculate the walking cost. The energetic cost of walking determined for the above two cases is then compared to the baseline cost. It is observed that addition of torsional springs at both hips reduce the walking cost up to $50 \%$, support hip up to $85 \%$ with spring stiffness as an optimization variable for both cases while mechanically locking the support knee reduces the cost of walking up to $25 \%$ with gait and knee locking angle optimized.
\end{abstract}

\section{INTRODUCTION}

In the past two decades, the studies on the passive robots have significantly attracted the attention of researchers to solve the problem of energy optimization and human like walking. A robot is called passive when no external energy (actuator) is required for walking. In 1990 McGeer [15] had first presented his work on passive dynamic walking and demonstrated that it is possible to exploit the mass distribution of the robot to make it walk on a shallow slope without actuation [9].

Based on the McGeer's work on passive walkers, research community of humanoid robots has developed passive dynamic walkers with minimal actuation to make them walk on flat surfaces [3], [4]. These robots are capable of walking on flat surfaces and have energy cost almost equal to that of the human. The three most famous level ground powered walking robots based on the ramp-walking design are the Cornell biped, the Delft biped (Denise) [2], [24] and the MIT learning biped [4]. These powered bipeds have motions close to those of their ramp-walking counterparts [4]. Gini et all [9] extended this idea to fully actuated robots and constructed their robot with joint compliance and special knee design to improve walking efficiency.

The humanoid robots are biologically inspired robots. They look like a human having two legs, torso and hands, although several types of bipedal robots may model only

This work is supported by ANR grants for the R2A2 project and by Higher Education Commission (HEC) of Pakistan under overseas scholarship program. part of the body. For example, most of the active dynamic walking bipeds in research laboratories have only two legs and a torso [20], [21], [7]. The number of humanoid robots having arms, head and feet are increasing and researchers are concentrating on the energetic effects of arms, feet and compliance in the walking gaits by adding springs to the bipeds. Most of the researchers including [6], [19], [20], [17] are motivated by the hypothesis that bipeds with compliant ankles may be able to exhibit more natural-looking gaits with better energetic efficiency and walking stability as compared to bipeds without compliant joints. Several researchers studied that the design of the knee joint can help to improve the walking efficiency [10] and others concentrated on the addition of passive elastic members in the knee and hip joints. The compliant swinging leg can reduce energetic cost by producing anti-gravity torques that lower the amount of actuator work required for leg swinging [16].

The most important and critical issue in the field of robotics especially in the humanoid robot's gait generation is the consumption of energy during walking. The researchers are trying to get bipeds to approximate the motions of human walking as closely as possible while being stable and efficient [3], [18], [25]. Studies show that the legs of the humanoid robots consume more energy in the stance phase than in the swing phase [5]. This difference in energy consumption is because of the demand of high torques to support the robot weight on the ground. Therefore, there is room for significant improvement in optimizing the energy consumption of the support leg. Förg [5] studied that the most inefficient (energy consuming) joint is the support knee joint. They introduced springs to reduce the consumption of energy.

Recently linear elastic members (springs) have been used to recover the lost energy, decrease the energy consumption and to stabilize the walking gait. In most of the cases, springs are added to the ankle of the biped to store energy and to use it when needed. This stored energy is mainly used during ankle push-off just before heel strike of free leg. Geyer et al. [8] introduced the idea of spring legs with compression springs for walking and running. They showed that compliant legs are essential to explain walking mechanics. They studied a bipedal spring-mass model which includes the double support as an essential part of its motion and reproduced the characteristics dynamics of walking. Their model combines the basic dynamics of walking and running in one mechanical system. In another study, compliant controller is used to tune the stiffness of the adaptable compliant actuator to reduce energy consumption of the biped Lucy during walking [23]. Lucy is powered by pleated pneumatic artificial muscles and 
is able to walk up to a speed of $0.15 \mathrm{~m} / \mathrm{s}$.

Studies indicate that there is a direct trade-off between the toe-off impulse from the trailing leg and the rotational torque between the legs [14]. Using the toe-off impulse alone to power the gait is four times less energetically expensive then using the hip torque alone [13], [14]. Another method of reducing energy consumption is to mechanically lock the support knee just before impact and release the lock at the end of double support phase. The knee-lock with active release mechanism is found to be technologically simple and energetically efficient [22]. However, neither the combined effects of knee locking and addition of springs have been explored nor the effects of compliance on energy consumption have been presented as a function of walking speed.

In this paper, we carried out four different studies, first on the robot with no springs and no locking mechanism, second with spring at different articulations, third with knee locked during support phase and fourth with the support knee locked and springs added to hip joints. We assume that the support knee can be mechanically locked and the torque required is provided by the mechanical lock. The mass of the locking mechanism is considered to be negligible. All the joint actuators are supposed to be frictionless and the torque and velocity discontinuities are allowed at impact. Similar kind of study has been done and presented for impactless walking of the biped [11].

Our simulated robot is composed of a trunk (including mass of arms), two thighs, two shins and two feet. The steps are considered to be cyclic having single support phases separated by flat foot impulsive impacts. The reference gait trajectories are defined by cubic spline functions of joint angles.

The paper is structured as follows: physical parameters of the robot and type of studies carried out are presented in section II, section III presents the dynamic model of the robot. In section IV optimization strategy with and without springs and with knee locking is explained. This section also presents the numerical study of the influence of springs and knee locking on the energy consumption of bipedal robot during walking at different walking speeds. Results are explained in section V while section VI gives the conclusion and perspectives.

\section{PRESENTATION OF THE ROBOT}

Our seven-link bipedal robot, presented in figure 1 , is a planar bipedal robot, composed of two identical legs and a torso. Each leg consists of a thigh, shin and a rigid flat foot. All joints are revolute, frictionless and can only move in the sagittal plane. The desired gait consists of single support phases separated by instantaneous and perfectly inelastic impact phase. Right and left feet are supposed to be the stance and swing foot respectively.

The walking step starts with a single support phase and ends with an impact on the swing foot where the feet exchange their role i.e. the stance foot becomes the swing foot and vice versa. The impact is considered to be flat

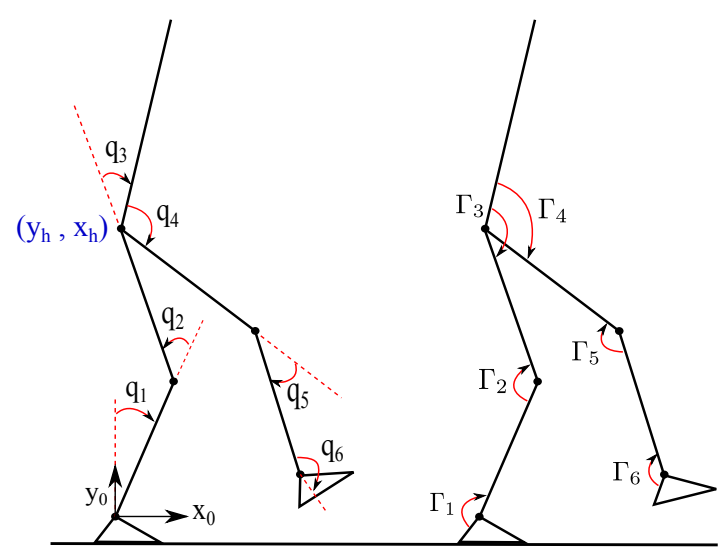

Fig. 1. Planar biped, generalized coordinates representation and applied torques

foot and there is no rotation on the support heel or toe during swing phase. Stance foot is considered as the base link $R_{0}$ of the robot. The physical parameters of the robot in $2 \mathrm{D}$ are presented in table I. These parameters are derived from the humanoid robot HYDROiD [1] having body mass and length similar to those of a human. The HYDROiD is in the manufacturing phase and will be available for experimentation in third quarter of this year.

We carried out four different types of studies which are explained below:

Case A. The robot's walking trajectories are optimized and the energetic cost of walking is calculated without adding springs or blocking the knee.

Case B. Springs are added to the hip, knee or ankle joints of the robot. These studies have the following two sub-cases:

Case B1. Spring is added only to the support leg joints (ankle, knee or hip).

Case B2. Identical springs are added to both joints of the ankle, knee or hip.

In all the cases where a spring is added to any of the joint, the spring stiffness coefficient $K$ is optimized along with the gait. The spring offset or bias angle $q_{s j}$ is kept zero which helps to keep the biped in vertical standing position and reduces the applied actuator's torque.

Case C. The knee of the stance leg is mechanically locked at impact and during the entire support phase without adding springs at any of the joint.

Case C1. The knee locking angle $(\beta)$ and the walking gait are optimized.

Case C2. Based on the numerical values obtained in case $\mathbf{C 1}$, a constant value of $\beta$ is selected and then the gait is optimized.

Case D. Support knee is mechanically locked and identical springs are added to both hip joints. The knee locking angle $\beta$ and spring stiffness $K$ are optimized along with the gait trajectory. 
TABLE I

PHySICAL PARAMETERS OF THE ROBOT

\begin{tabular}{|c|l|c|c|c|c|}
\hline Link & Description & $\begin{array}{c}\text { Mass } \\
K g\end{array}$ & $\begin{array}{c}\text { Length } \\
(\mathrm{m})\end{array}$ & $\begin{array}{c}\text { Gravity Center } \\
(\mathrm{m})\end{array}$ & $\begin{array}{c}\text { Inertia } \\
\mathrm{Kg} \cdot \mathrm{m}^{2}\end{array}$ \\
\hline \hline 0 & Support Foot & 0.678 & $\begin{array}{c}L_{p}=0.20700 \\
h_{p}=0.06425\end{array}$ & $\begin{array}{c}S p_{x}=0.01350 \\
S p_{y}=0.03213\end{array}$ & 0.00175 \\
\hline 1 & Support Shin & 2.188 & 0.392 & 0.16856 & 0.02765 \\
\hline 2 & Support Thigh & 5.025 & 0.392 & 0.16856 & 0.06645 \\
\hline 3 & Trunk & 29.27 & 0.5428 & 0.192065 & 0.81496 \\
\hline 4 & Swing Thigh & 5.025 & 0.392 & 0.16856 & 0.06645 \\
\hline 5 & Swing Shin & 2.188 & 0.392 & 0.16856 & 0.02765 \\
\hline 6 & Swing Foot & 0.678 & $\begin{array}{c}L_{p}=0.20700 \\
h_{p}=0.06425\end{array}$ & $\begin{array}{c}S p_{x}=0.01350 \\
S p_{y}=0.03213\end{array}$ & 0.00175 \\
\hline
\end{tabular}

\section{MODEL OF THE ROBOT}

In this section, the dynamic and impact models for the biped under study are established and discussed in detail for the four cases presented in Section II. Our dynamic model is based on the assumption of flat foot contact with the ground, which means that the support foot does not rotate during the swing phase and the swing foot touches the ground with flat contact at the end of the swing phase.

\section{A. Dynamic Model in Single Support}

The robot configuration in single support phase can be described by the reduced position vector $q=$ $\left[\begin{array}{llllll}q_{1} & q_{2} & q_{3} & q_{4} & q_{5} & q_{6}\end{array}\right]^{\mathrm{t}}$. This representation is shown in figure 1 . Here, $\Gamma=\left[\begin{array}{llllll}\Gamma_{1} & \Gamma_{2} & \Gamma_{3} & \Gamma_{4} & \Gamma_{5} & \Gamma_{6}\end{array}\right]^{\mathrm{t}}$ is the joint torque vector and $R_{j}=\left[\begin{array}{ll}R_{x} & R_{y}\end{array}\right]^{\mathrm{t}}$ contains the ground reaction forces on foot $j$.

The dynamic model can be developed by using the Lagrange formulation. In single support phase, the dynamic model, considering an implicit liaison of the stance foot with the ground, i.e. there is no take off and no sliding during the single support phase, can be written:

$$
\mathbf{A}(\mathbf{q}) \ddot{\mathbf{q}}+\mathbf{C}(\mathbf{q}, \dot{\mathbf{q}}) \dot{\mathbf{q}}+\mathbf{G}(\mathbf{q})=\boldsymbol{\Gamma}
$$

where $\mathbf{A}(q) \in \mathbb{R}^{6 \times 6}$ is the positive definitive inertia matrix, $\mathbf{C}(q, \dot{q}) \in \mathbb{R}^{6 \times 6}$ contains the Coriolis and centrifugal forces, $\mathbf{G}(q) \in \mathbb{R}^{6 \times 1}$ is the vector of gravity forces. The ground reaction forces acting on the feet can be calculated by applying the dynamic equilibrium principle at the center of mass of the robot.

$$
\left[\begin{array}{l}
R_{1 x} \\
R_{1 y}
\end{array}\right]+\left[\begin{array}{l}
R_{2 x} \\
R_{2 y}
\end{array}\right]=m\left[\begin{array}{l}
\ddot{x}_{g} \\
\ddot{y}_{g}
\end{array}\right]+m\left[\begin{array}{l}
0 \\
g
\end{array}\right]
$$

Here $m$ is the biped's mass, $\ddot{x}_{g}$ and $\ddot{y}_{g}$ are the horizontal and vertical components of the biped's center of mass respectively, $R_{j x}$ and $R_{j y}$ are the horizontal and vertical components of the ground reaction force on the foot $j$ respectively. In single support phase, the ground reaction force $R_{2}$ on the swing foot is zero which enables us to calculate the reaction force $R_{1}$ on the stance foot by using equation (2).

\section{B. Dynamic Model in Double Support}

The dynamic model in double support phase can be written to take into account the reaction forces applied by ground on the robot. For the definition of the dynamic model in double support, we use spatial coordinate system with explicit liaison. Hence the state of the robot can be represented by the position vector $q_{e}=\left[\begin{array}{lllllllll}q_{0} & q_{1} & q_{2} & q_{3} & q_{4} & q_{5} & q_{6} & x_{h} & y_{h}\end{array}\right]^{\mathrm{t}}$. The two Cartesian coordinates $x_{h}, y_{h}$ are the abscissa and the ordinate of the hip joint and $q_{0}$ is the support foot angle with horizontal axis. The dynamic model in double support is

$$
\mathbf{A}\left(\mathbf{q}_{e}\right) \ddot{\mathbf{q}}_{e}+\mathbf{C}\left(\mathbf{q}_{e}, \dot{\mathbf{q}}_{e}\right) \dot{\mathbf{q}}_{e}+\mathbf{G}\left(\mathbf{q}_{e}\right)=\mathbf{B} \boldsymbol{\Gamma}+\mathbf{J}_{1}^{\mathrm{t}} \mathbf{R}_{1}+\mathbf{J}_{2}^{\mathrm{t}} \mathbf{R}_{2}
$$

where $\mathbf{A}\left(\mathbf{q}_{e}\right) \in \mathbb{R}^{9 \times 9}$ is the positive definitive inertia matrix, $\mathbf{C}\left(\mathbf{q}_{e}, \dot{\mathbf{q}}_{e}\right) \in \mathbb{R}^{9 \times 9}$ represents the vector of Coriolis and centrifugal forces, $\mathbf{G}\left(\mathbf{q}_{e}\right) \in \mathbb{R}^{9 \times 1}$ contains the gravity forces, $\mathbf{B} \in \mathbb{R}^{9 \times 9}$ is the actuation matrix consisting of zeros and ones, $\mathbf{J}_{1}^{\mathrm{t}} \in \mathbb{R}^{3 \times 9}$ and $\mathbf{J}_{2}^{\mathrm{t}} \in \mathbb{R}^{3 \times 9}$ are the Jacobian matrices of foot 1 and 2 respectively and $\mathbf{R}_{1} \in \mathbb{R}^{3 \times 1}$ and $\mathbf{R}_{2} \in \mathbb{R}^{3 \times 1}$ are the vectors of ground reaction forces and moment on foot 1 and 2 respectively.

\section{The Impact Model}

As we are considering impulsive impact, the support foot will immediately leave the ground after having impact on swing foot (foot 2). Therefore the term $\mathbf{J}_{1}^{\mathbf{t}} \mathbf{R}_{1}=0$ and the equation (3) can be rewritten in the following form.

$$
\mathbf{A}\left(\mathbf{q}_{e}\right) \ddot{\mathbf{q}}_{e}+\mathbf{C}\left(\mathbf{q}_{e}, \dot{\mathbf{q}}_{e}\right) \dot{\mathbf{q}}_{e}+\mathbf{G}\left(\mathbf{q}_{e}\right)=\boldsymbol{\Gamma}+\mathbf{J}_{2}^{\mathrm{t}} \mathbf{R}_{2}
$$

Now the following constraints can be added by using the Jacobian of foot 2 .

$$
\mathbf{J}_{2} \ddot{\mathbf{q}}_{e}+\frac{d \dot{\mathbf{J}}_{2}}{d t} \dot{\mathbf{q}}_{e}=0
$$

The impact model is deduced from the dynamic model in double support by assuming that the acceleration of the robot and the reaction force are Dirac delta-functions. The impact model is derived from equation (4) and is written in the following form:

$$
\mathbf{A}\left(\mathbf{q}_{e}\right)\left(\dot{\mathbf{q}}_{e}^{+}-\dot{\mathbf{q}}_{e}^{-}\right)=\mathbf{J}_{2}^{\mathrm{t}} \mathbf{I}_{2}
$$

Where $\dot{\mathbf{q}}_{e}^{-}$and $\dot{\mathbf{q}}_{e}^{+}$are the vectors of links velocities before and after impact respectively. Vector $\mathbf{I}_{2} \in \mathbb{R}^{3 \times 1}$ represents the impulsive ground reaction forces and moment on the support foot. The velocity of the swing foot (new support foot) after impact is zero and this constraint is expressed as:

$$
\mathbf{J}_{2} \dot{\mathbf{q}}_{e}^{+}=0
$$

The matrix equations (6) and (7) are simultaneously solved to find the velocities vector $\dot{q}_{e}^{+}$just after impact and the impact impulsive forces and moment vector $\mathbf{I}_{2} \in \mathbb{R}^{3 \times 1}$.

$$
\left[\begin{array}{cc}
\mathbf{A} & -\mathbf{J}_{2}^{\mathrm{t}} \\
\mathbf{J}_{2} & 0
\end{array}\right]\left[\begin{array}{c}
\dot{\mathbf{q}}_{e}^{+} \\
\mathbf{I}_{2}
\end{array}\right]=\left[\begin{array}{c}
\mathbf{A} \dot{\mathbf{q}}_{e}^{-} \\
0
\end{array}\right]
$$


The above system of equations is based on the following hypothesis:

1) The impact is inelastic and instantaneous

2) The swing leg hits the ground with flat foot and the support leg leaves the ground immediately after impact

3) The robot configuration is constant during impact

4) The velocities, accelerations and torques are discontinuous at impact

\section{The Impact Model with Knee Locked}

We suppose that the knee of the robot can be locked mechanically at any desired or pre-selected position. The knee locking mechanism is assumed to be weightless and its energy consumption is negligible. The locking is bilateral and the torque at knee joint is provided by the mechanical lock. The knee is locked just at impact and remains locked until the other foot (swing) comes in contact with the ground. At this point, the previously locked knee is released and the new support knee is locked.

The knee locking at impact modifies the impact model in equation (6) and the modified impact model is

$$
\mathbf{A}\left(\mathbf{q}_{e}\right)\left(\dot{\mathbf{q}}_{e}^{+}-\dot{\mathbf{q}}_{e}^{-}\right)=\mathbf{J}_{2}^{\mathrm{t}} \mathbf{I}_{2}+\mathbf{J}_{k}^{\mathrm{t}} \mathbf{I}_{k}
$$

where $\mathbf{I}_{k}$ is the impulsive reaction on the locked knee and $\mathbf{J}_{k} \in \mathbb{R}^{1 \times 9}$ represents the Jacobian of the locked knee. The velocity of the knee joint after impact must be zero and for this we impose the following constraint equation

$$
\mathbf{J}_{k} \dot{\mathbf{q}}_{e}^{+}=0
$$

The matrix equations (7), (9) and (10) are simultaneously solved to find the velocities vector $\dot{\mathbf{q}}_{e}^{+}$just after impact, the impact impulsive forces and moment vector $\mathbf{I}_{2} \in \mathbb{R}^{3 \times 1}$ of the support foot and the knee impulse $I_{k}$. The system of equation is written in matrix form as below:

$$
\left[\begin{array}{ccc}
\mathbf{A} & -\mathbf{J}_{2}^{\mathrm{t}} & -\mathbf{J}_{k}^{\mathrm{t}} \\
\mathbf{J}_{2} & 0 & 0 \\
\mathbf{J}_{k} & 0 & 0
\end{array}\right]\left[\begin{array}{c}
\dot{\mathbf{q}}_{e}^{+} \\
\mathbf{I}_{2} \\
\mathbf{I}_{k}
\end{array}\right]=\left[\begin{array}{c}
\mathbf{A} \dot{\mathbf{q}}_{e}^{-} \\
0 \\
0
\end{array}\right]
$$

\section{E. Dynamic Model with Springs}

The goal of our work is to improve the energy efficiency of the bipedal robot under study and for this purpose, we added torsional springs to the robot structure in parallel with the existing actuators. These springs helped to reduce the actuator torque and consequently the energy consumption of the biped. The dynamic model (1) with elastic elements becomes

$$
\mathbf{A}(\mathbf{q}) \ddot{\mathbf{q}}+\mathbf{C}(\mathbf{q}, \dot{\mathbf{q}}) \dot{\mathbf{q}}+\mathbf{G}(\mathbf{q})+\Gamma_{s}=\Gamma
$$

Where $\Gamma_{s}$ is the vector of spring torque and is obtained from following equation.

$$
\boldsymbol{\Gamma}_{s}=\sum_{j=1}^{n} \boldsymbol{\Gamma}_{s j}
$$

Where $j$ is the joint on which spring is installed, $n$ is the total number of joints and $\boldsymbol{\Gamma}_{s j}$ is the spring torque provided by joint $j$. The spring torque is obtained from derivative of the spring potential energy. The potential energy of the spring is given by:

$$
U_{j}=\frac{1}{2} K_{j}\left(q_{j}-q_{s j}\right)^{2}
$$

Where $U_{j}$ and $K_{j}$ are the spring potential energy and spring stiffness respectively at joint $j, q_{j}$ is the angle between link $j$ and $j-1$ and $q_{s j}$ is the spring offset or bias angle at joint $j$.

According to Lagrange formulation, to calculate the spring torque vector $\boldsymbol{\Gamma}_{s j}$ on $j^{\text {th }}$ joint, we have

$$
\Gamma_{s j}=\frac{\delta U_{j}}{\delta q}
$$

\section{F. The Zero Moment Point (ZMP) of the Biped}

To avoid the rotation of the support foot, the ZMP of the biped must be located in the supporting foot area. The position of the ZMP is the point on the ground such that the moment exerted by the ground is zero along the ankle axis. The foot geometry of our biped is presented in figure 2 and from the balance of forces on ankle axis, the $Z M P_{x}$ in single support phase is calculated from equation (16).

$$
Z M P_{x}=\frac{\Gamma+S p_{x} m_{p} g-h_{p} R_{x}}{R_{y}}
$$

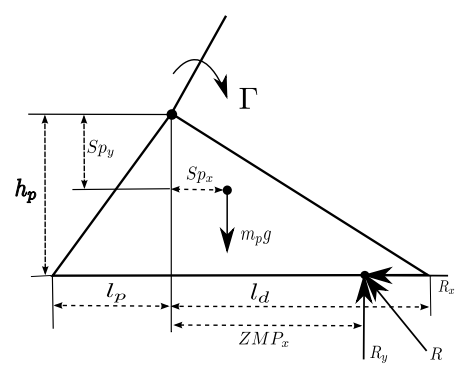

Fig. 2. Biped foot geometry ( $m_{p}$ is the foot mass, $g$ is gravity and $S p$ is foot mass center)

\section{TRAJECTORY OPTIMIZATION}

In optimization problem, reference trajectory for a walking step is generated and then optimized to minimize a criterion under constraints. We are using a polynomial of order four for the evolution of actuated joints motion as a function of time to generate reference trajectory. A fourth degree polynomial ensures that the jerk of the joints motion is continuous. To reduce the number of optimization parameters, we consider the reference trajectory to be cyclic. The trajectory is repeated after every walking step. The reference polynomial function is represented below:

$$
q_{i}(t)=\sum_{k=0}^{4} a_{k} t^{k} \quad \text { for } \mathrm{i}=1 \text { to } 6
$$


To determine the coefficients of equation (17), five boundary conditions are needed. These boundary conditions are the initial joint configuration $q_{i n i}$, velocities $\dot{q}_{i n i}$ at time zero, the intermediate configuration $q_{\text {int }}$ at time $T / 2$, the final joint configuration $q_{f i n}$, and velocities $\dot{q}_{f i n}$ at time $T$.

\section{A. The Optimization Parameters}

The optimization parameters of the robot are presented in this section. These parameters are optimized using the parametric optimization procedure to find the optimal solution by minimizing the predefined optimization criterion. The Sequential Quadratic Programing (SQP) method [10] is used to optimize the reference trajectory using the Matlab fmincon function. We need to define the criterion (objective function) to minimize, the set of parameters to optimize, and the constraints the robot must satisfy during walking. The optimization parameters used in our study are

- 4 parameters just before impact (i.e. hip configuration $\left(h_{x}, h_{y}\right)$, trunk angle around horizontal axis and step length $d$ ).

- 6 parameters of final velocities just before impact. Initial velocities (velocities just after impact) can be found by impact model.

- 6 parameters of intermediate configuration of the robot.

The walking speed is manually selected and the step time $T$ is calculated from distance traveled $d$ (step length) and the selected speed.

\section{B. The Optimization Criterion}

The criterion we used is based on the actuator's torque. This criterion is used to optimize the trajectory, which minimizes the actuators effort to take one step i.e. cover the distance $d$ for a motion on a half cycle of duration $T$.

$$
C_{\Gamma}=\frac{1}{d} \int_{0}^{T} \Gamma^{\mathrm{t}} \Gamma \mathrm{d} t
$$

Where $C_{\Gamma}$ is the objective function to minimize and $\boldsymbol{\Gamma}$ represents the joints torque matrix. For electrical DC motors $R I^{2}$, which is proportional to $\Gamma^{2}$ are the losses by Joule effect where $R$ and $I$ are the motor resistance and current respectively.

Our objective is to minimize the optimization criterion $C_{\Gamma}$ by finding the optimal values of optimization parameters $P_{0}$ under non-linear constraints and polynomial functions of degree four as basis of motion. The optimization problem is formulated as follows

$$
\begin{cases}\text { Minimize } & C_{\Gamma}\left(P_{0}\right) \\ \text { Subject to } & g_{j}\left(P_{0}\right) \leq 0, j=1,2, \ldots . ., l\end{cases}
$$

Where $C_{\Gamma}\left(P_{0}\right)$ is the objective function to minimize with $l$ constraints $g_{j}\left(P_{0}\right) \leq 0$ to satisfy. These constraints are defined in the following section. For our parametric optimization problem, we have sixteen parameters to optimize for our robot trajectory without addition of springs.

\section{The Optimization Constraints}

To ensure that the biped will successfully walk and the trajectory is possible, a number of constraints must be satisfied. Two types of constraints are applied to ensure walking on level ground.

1) Basic Constraints: These constraints are required for successful walking of the biped and to ensure that the generated trajectory is possible and valid.

The vertical component of the Ground Reaction Forces (GRF) on stance foot must always be positive so that the robot's foot remains on the ground all the time during walking step. The support foot must not slip during walking after selecting a suitable value of coefficient of friction $\mu$.

$$
\left\{\begin{array}{l}
R_{y}>0 \\
\mu R_{y} \geq\left|R_{x}\right|
\end{array}\right.
$$

here $R_{y}$ and $R_{x}$ are the vertical and horizontal components of reaction force respectively.

The ZMP of the biped must be within the interior of the support polygon.

$$
l_{p} \leq Z M P_{x} \leq l_{d}
$$

here $l_{p}$ is the foot length between heel and ankle and $l_{d}$ is the length from toe to ankle see figure 2 .

The swing foot must not touch the ground during swing phase i.e. the distance of swing foot's heel and toe must be positive.

$$
\left\{\begin{array}{l}
y_{\text {heel }}>0 \\
y_{\text {toe }}>0
\end{array}\right.
$$

here $y_{\text {heel }}$ and $y_{\text {toe }}$ are the vertical distances of swing foot heel and toe respectively during swing phase.

The heel and toe velocities of the foot leaving the ground just after impact must be positive to ensure takeoff (23) and the foot coming in contact with ground must not slip or bounce back during impact (24).

$$
\begin{gathered}
\left\{\begin{array}{l}
V_{\text {heel }} \geq 0 \\
V_{\text {toe }} \geq 0
\end{array}\right. \\
\left\{\begin{array}{l}
I_{y}>0 \\
\mu I_{y} \geq\left|I_{x}\right|
\end{array}\right.
\end{gathered}
$$

2) Technological Constraints: These constraints consist of physical limitations of the robot's actuators and articulations. Each actuator can produce a limited torque and velocity. The upper and lower joint configuration limits must also be satisfied.

$$
\left\{\begin{array}{l}
\left|\Gamma_{i}\right|-\Gamma_{i, \max } \leq 0, \quad \text { for } \quad i=1, \ldots, 6 \\
\left|\dot{q}_{i}\right|-\dot{q}_{i, \max } \leq 0, \quad \text { for } i=1, \ldots, 6 \\
q_{i, \min } \leq q_{i} \leq q_{i, \max }, \quad \text { for } i=1, \ldots, 6
\end{array}\right.
$$

Where $\Gamma_{i, \max }$ and $\dot{q}_{i, \max }$ represents the maximum value of torque and velocity respectively for each actuator and $q_{i, \min }$ and $q_{i, \max }$ are the minimum and maximum joint position limits respectively. 


\section{RESULTS}

In this section we present the simulation results obtained for all the four cases presented in Section II. We compare the optimization criteria obtained for all cases with that of our basic robot in case $\mathbf{A}$ and then present the percentage of energy saved in each case. The data points are joined using fourth order polynomial interpolation to have smooth curves.

Our results show that the most effective way to reduce energy consumption during walking is to add springs to the support joints only and the most economical is the hip joint (see fig. 3) which economizes up to $85 \%$ of energy at 0.85 $\mathrm{m} / \mathrm{sec}$ as presented in figure 4 .

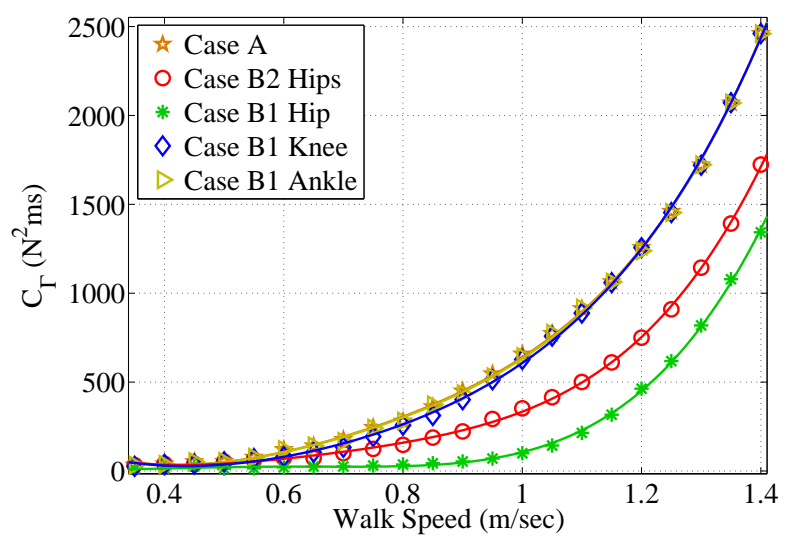

Fig. 3. Value of objective function as a function of walking speed

Adding springs to the support leg is possible by having a variable spring stiffness mechanism [25] capable of producing spring stiffness from zero to required value, however this mechanism will add an additional mass and the robot's parameters will change. The second version of AwAS (A new Actuator with Adjustable Stiffness) is a light weight actuator, whose stiffness can be tuned from zero to rigid [12].

In our simulation tests, we assumed that this mechanism is massless and the energy consumed by the mechanism is negligible. To avoid this variable stiffness mechanism, we restrained ourselves to the simple option and studied the effects of adding identical torsional springs to both legs.

Figure 3 presents the evolution of criterion for our robot in case $\mathbf{A}$, case $\mathbf{B} 1$ where spring was introduced to the support knee and then to support hip and case $\mathbf{B} 2$ where identical springs were added to both hip joints. Figure 3 shows that the optimization criterion is significantly reduced after introducing identical springs to both hip joints in parallel with existing actuator. It was however observed that adding springs to both knees or ankles were not effective in our case contrary to [19], [24] where they found ankle springs useful for stability and energetic efficiency. This is because we considered a flat foot impact and there is no double support phase and no rotation of support foot during entire swing phase. In addition we kept the spring offset angle zero which can improve the role of ankle springs if tuned properly.

Figure 4 gives evolution of percentage economy as a function of walking speed corresponding to figure 3. The

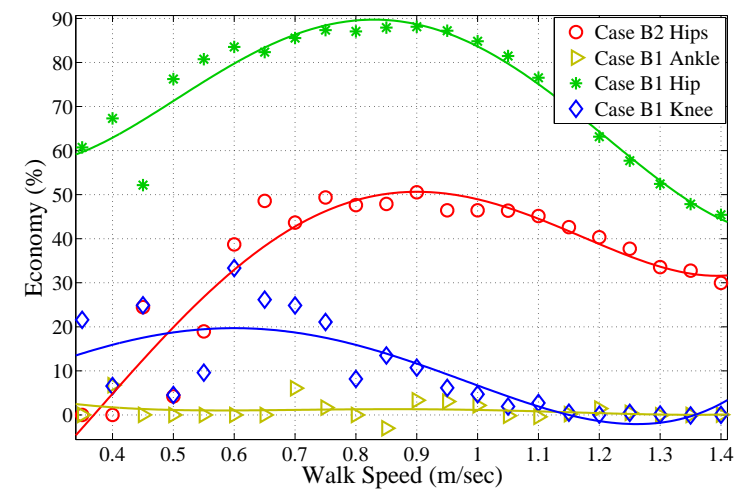

Fig. 4. Percentage energy saving as a function of walking speed

criterion was reduced to $50 \%$ by adding identical springs to hip joints and maximum energetic efficiency was obtained at walking speeds from $0.7 \mathrm{~m} / \mathrm{sec}$ to $1.0 \mathrm{~m} / \mathrm{sec}$.

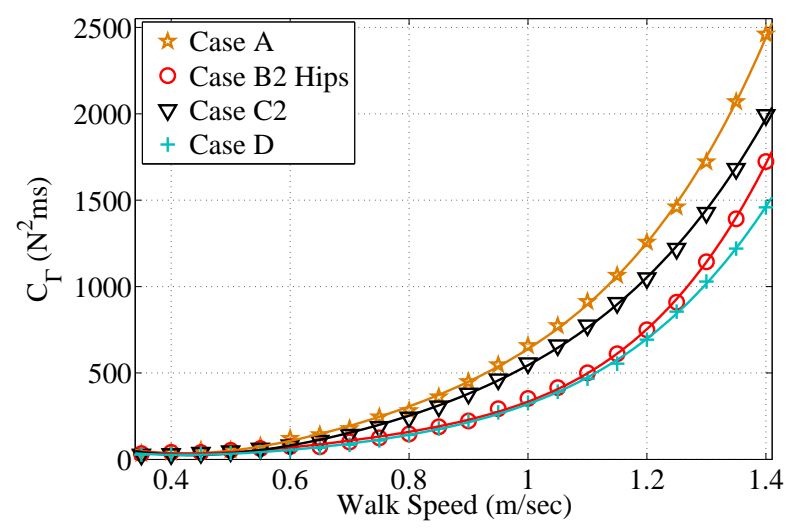

Fig. 5. Value of objective function as a function of walking speed

Figure 5 gives the evolutions of selected criterion as a function of walking speed for our biped in case $\mathbf{A}$, case $\mathbf{B 2}$ where identical springs were added to both hip joints, case C2 where knee locking angle $\beta$ of 1 degree was obtained from case $\mathbf{C 1}$ and for a biped in case $\mathbf{D}$ with an identical spring on both hips and support knee locked. The energy consumption for a biped with support knee locked and springs on both hips is always less than that of a biped with only knee locking or hip springs.

The evolution of percentage energy saved is represented in figure 6 which shows that the combined energetic efficiency of locking support knee and adding identical torsional springs to both hips is always greater than the individual effect of locking knee or adding springs to both hips.

\section{CONCLUSION AND PERSPECTIVES}

In this paper we studied a seven-link bipedal robot with three different strategies, first by adding torsional springs to different joints, second by mechanically locking the knee and third by combining the two strategies i.e. locking the support knee and adding identical springs to the hip joints 


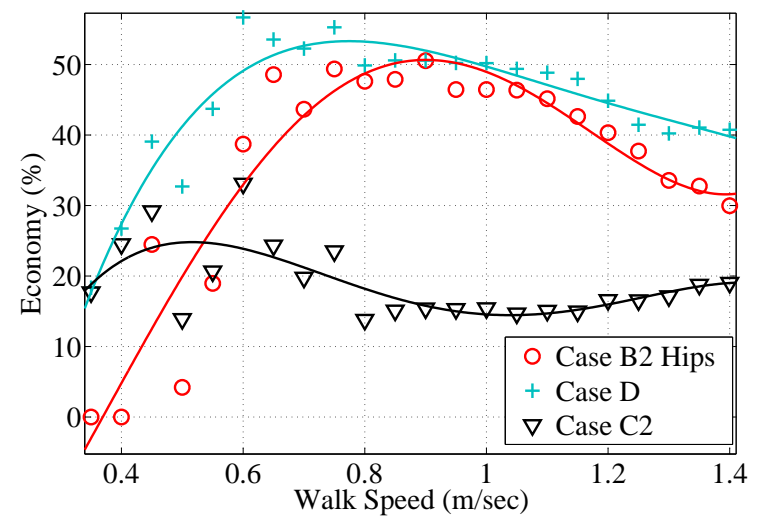

Fig. 6. Percentage energy saving as a function of walking speed

which give the maximum energetic economy. The main focus of the study was to economize the energy consumption of a bipedal robot during walking.

We conclude from this study that the energy consumption of a biped during walking is significantly reduced by adding identical torsional springs at the hip joints and mechanically locking the knee. The energetic efficiency of blocking the knee alone or adding springs to the hip joints only is also noticeable. This strategy will significantly improve the energetic efficiency as well as the autonomy of our biped.

In line with the previous research, our study reinforces the idea of using passive joint stiffness to improve energetic efficiency of the biped especially on the hip joints in our case. However, contrary to previous work, ankle springs were not effective in our study.

In perspective of this study, the next step is to explore the effects of spring offset or bias angle and the ankle springs with foot rotation on the energetic efficiency of the biped. A more complex walking gait with foot rotation and double support phase can also be studied. This can also be extended to study effects on a 3D bipedal robot.

\section{REFERENCES}

[1] S. Alfayad, F.B. Ouezdou, and F. Namoun. New three dof ankle mechanism for humanoid robotic application: Modeling, design and realization. In proceedings of the 2009 IEEE/RSJ International Conference on Intelligent Robots and Systems (IROS 2009), pages 4969-4976, 2009.

[2] S.O. Anderson, M. Wisse, C.G. Atkeson, J.K. Hodgins, G.J. Zeglin, and B. Moyer. Powered bipeds based on passive dynamic principles. In proceedings of the 2005 5th IEEE-RAS International Conference on Humanoid Robots, pages 110-116, 2005.

[3] S.H. Collins and A. Ruina. A bipedal walking robot with efficient and human-like gait. In proceedings of the 2005 IEEE International Conference on Robotics and Automation (ICRA 2005), pages 19831988, 2005.

[4] Steve Collins, Andy Ruina, Russ Tedrake, and Martijn Wisse. Efficient bipedal robots based on passive-dynamic walkers. Science, 307(5712):1082-1085, 2005.

[5] Heinz Ulbrich Daniela Förg, Martin Förg. A bipedal robot model with elastic actuation. In proceedings of the 1st Joint International Conference on Multibody System Dynamics, May 25-27 2010.

[6] K.D. Farrell, C. Chevallereau, and E.R. Westervelt. Energetic effects of adding springs at the passive ankles of a walking biped robot. In proceedings of the IEEE International Conference on Robotics and Automation (ICRA 2007), pages 3591-3596, 2007.
[7] Y. Fujimoto. Minimum energy biped running gait and development of energy regeneration leg. In proceedings of the 8th IEEE International Workshop on Advanced Motion Control (AMC '04), pages 415-420, 2004.

[8] Hartmut Geyer, Andre Seyfarth, and Reinhard Blickhan. Compliant leg behaviour explains basic dynamics of walking and running. In proceedings of the Royal Society B, 2006.

[9] G. Gini, U. Scarfogliero, and M. Folgheraiter. Human-oriented biped robot design: Insights into the development of a truly anthropomorphic leg. In proceedings of the IEEE International Conference on Robotics and Automation (ICRA '07), pages 2910-2915, 2007.

[10] A. Hamon and Y. Aoustin. Study of different structures of the knee joint for a planar bipedal robot. In proceedings of the 9th IEEE-RAS International Conference on Humanoid Robots (Humanoids 2009), pages $113-120,2009$.

[11] A. Haq, Y. Aoustin, and C. Chevallereau. Compliant joints increase the energy efficiency of bipedal robot. In The 14th International Conference on Climbing and Walking Robots and the Support Technologies for Mobile Machines (CLAWAR2011), september 6-8 2011.

[12] Amir Jafari, Nikos G. Tsagarakis, and Darwin G. Caldwell. Awas-ii: A new actuator with adjustable stiffness based on the novel principle of adaptable pivot point and variable lever ratio. In proceedings of the 2011 IEEE International Conference on Robotics and Automation (ICRA), pages 4638-4643, 2011.

[13] Arthur D. Kuo. Energetics of actively powered locomotion using the simplest walking model. Journal of Biomechanical Engineering, 124(1):113-120, 2002.

[14] Cara L. Lewis and Daniel P. Ferris. Walking with increased ankle pushoff decreases hip muscle moments. Journal of Biomechanics, 41(10):2082-2089, 2008.

[15] Tad McGeer. Passive dynamic walking. The International Journal of Robotics Research, 9(2):62-82, 1990.

[16] Shane Migliore, Lena Ting, and Stephen DeWeerth. Passive joint stiffness in the hip and knee increases the energy efficiency of leg swinging. Autonomous Robots, 29:119-135, 2010.

[17] Y. Nakano, K. Chono, K. Yoneda, and H. Kameishi. A dynamic biped walking robot based on the momentum mechanism with flexible beams. In proceedings of the IEEE/RSJ/GI International Conference on Intelligent Robots and Systems '94. 'Advanced Robotic Systems and the Real World', IROS '94, volume 2, pages 1318-1323 vol.2, 1994.

[18] Xiuhua Ni, Weishan Chen, and Junkao Liu. A comparison between human walking and passive dynamic walking. In proceedings of the 4th IEEE Conference on Industrial Electronics and Applications (ICIEA 2009), pages 2552-2555, 2009.

[19] T. Schauss, M. Scheint, M. Sobotka, W. Seiberl, and M. Buss. Effects of compliant ankles on bipedal locomotion. In proceedings of the IEEE International Conference on Robotics and Automation (ICRA '09), pages 2761-2766, 2009.

[20] M. Scheint, M. Sobotka, and M. Buss. Compliance in gait synthesis: Effects on energy and gait. In proceedings of the 8th IEEE-RAS International Conference on Humanoid Robots, Humanoids 2008, pages 259-264, 2008.

[21] F.M. Silva and J.A. Tenreiro Machado. Energy analysis during biped walking. In proceedings of the 1999 IEEE International Conference on Robotics and Automation (ICRA '09), volume 1, pages 59-64, 1999.

[22] K. Trifonov and S. Hashimoto. Active knee-lock release for passivedynamic walking machines. In proceedings of the IEEE International Conference on Robotics and Biomimetics (ROBIO 2007), pages 958963, 2007.

[23] Bram Vanderborght, Björn Verrelst, Ronald Van Ham, Michaël Van Damme, Pieter Beyl, and Dirk Lefeber. Development of a compliance controller to reduce energy consumption for bipedal robots. Autonomous Robots, 24(4):419-434, 052008.

[24] M. Wisse, D.G.E. Hobbelen, R.J.J. Rotteveel, S.O. Anderson, and G.J. Zeglin. Ankle springs instead of arc-shaped feet for passive dynamic walkers. In proceedings of the 6th IEEE-RAS International Conference on Humanoid Robots, pages 110-116, 2006.

[25] J. Yamaguchi, D. Nishino, and A. Takanishi. Realization of dynamic biped walking varying joint stiffness using antagonistic driven joints. In Proceedings of the IEEE 1998 IEEE International Conference on Robotics and Automation, volume 3, pages 2022-2029 vol.3, 1998. 